\title{
The Prevalence of Premenstrual Syndrome and the Influential Factors in Operating Room Technologists
}

\author{
Sedigheh Hannani ${ }^{1}$, Nasrin Ghanbary Nekoo $^{2}$, Fariba Nasiri Ziba ${ }^{3}$, Agha Fatemeh Hosseini ${ }^{4}$
}

\begin{abstract}
Background \& Aims: Premenstrual syndrome (PMS) is a common disorder of the reproductive age, which occurs periodically in the luteal phase of the menstrual cycle and has various physical, behavioral, and psychological symptoms, such as bloating, acne, back pain, depression, anxiety, and panic attacks. These symptoms decrease by the end of menstruation and disappear to some extent. In addition, the symptoms vary in different individuals, while they commonly manifest in menstrual cycles and may adversely affect familial and professional relations, productivity, and social activities. PMS symptoms may also reduce job efficiency in various professional groups and increase absenteeism, thereby leading to significant economic losses. Due to the effects of PMS on the performance of individuals in the workplace, as well as the challenging duties of female operating room technologists and frequent stressful situations in their work environment, the present study aimed to determine the prevalence of PMS and its influential factors in operating room technologists.

Materials \& Methods: This cross-sectional study was conducted on 112 operating room technologists employed in the teaching hospitals affiliated to Iran University of Medical Sciences during 2016-2017. Due to the limited sample population, the participants were selected via census sampling. Data were collected using three questionnaires, including a demographic questionnaire (validity confirmed by several professors of Iran University of Medical Sciences) and the premenstrual symptom screening questionnaire (Cronbach's alpha of 0.9, content validity ratio and content validity index of 0.7 and 0.8 , respectively by Siah Bazi [2011]). The latter consisted of 19 items and two sections; the first sections contains 14 indicators of mood and physical/behavioral symptoms, and the second section measures the impact of these symptoms on the life of the respondents with five items. Another data collection tool was the occupational stress questionnaire by Kahn. Rasouli (2005) obtained the reliability of this scale at the Cronbach's alpha of 0.79 , and the content validity was also confirmed based on the opinions of supervisors and consultants. In our study, the reliability of this tool was confirmed by calculating the Cronbach's alpha coefficient on 20 operating room technologists outside the research environment. Based on the obtained coefficient $(\mathrm{R}=0.75)$, the reliability of the tool was confirmed. In addition, the content validity was assessed and confirmed by 10 professors of the medical universities in Tehran. This questionnaire has 15 items, which are scored based on a five-point Likert scale. Scores of 15-30 indicate low occupational stress, scores of 30-45 show moderate occupational stress, scores of $>45$ show high occupational stress. After obtaining the required permit from the Ethics Committee of Iran University of Medical Sciences, receiving a letter of introduction, obtaining the permission of the research units (teaching hospitals of Iran University of Medical Sciences), and receiving informed consent from the participants, the questionnaires were distributed among the selected operating room technologists. Data analysis was performed in SPSS version 23 using descriptive statistics (frequency distribution table, central indices, and dispersion) and inferential statistics (Fisher's exact test, Chi-square).
\end{abstract}

\footnotetext{
1. Lecturer, Department of Operational Room Technology, School of Paramedical Sciences, Iran University of Medical Sciences, Tehran, Iran

2. MS in Operational Room Technology, School of Paramedical Sciences, Iran University of Medical Sciences, Tehran, Iran (Corresponding author) Tel: 02186704726 Email: ghanbarynekoo@yahoo.com

${ }^{3}$. Lecturer, Medical Surgical Department, School of Nursing and Midwifery, Iran University of Medical Sciences, Tehran, Iran

${ }^{4}$. Lecturer, Department of Biostatistics, School of Health, Iran University of Medical Sciences, Tehran, Iran
} 
Results: In total, $57.1 \%$ of the technologists had moderate-to-severe PMS, and $42.9 \%$ were not diagnosed with PMS or had mild PMS. No significant correlations were observed between age, body mass index, education level, physical exercise, and regular exercise with PMS. The highest prevalence of PMS was observed in the technologists aged less than 25 years, overweight subjects, and those without exercise/irregular exercise. However, significant correlations were denoted between PMS and the type of physical exercise (endurance/resistance), so that the prevalence of PMS was lower in the technologists with endurance training compared to those with resistance training or both $(\mathrm{P}=0.027)$. In addition, a significant association was observed between PMS and occupational stress, so that increased occupational stress would increase the incidence of the syndrome $(\mathrm{P}=0.005)$.

Coclusion: According to the results, PMS had a comparatively lower prevalence in the technologists with less occupational stress and those with physical exercise (especially endurance training). Given the importance of health, occupational quality, and the key role of technologists in the operating team, it is recommended that proper training classes and group counseling be provided to reduce occupational stress and increase the accuracy, skills, and knowledge of task performing. Furthermore, technologists should be encouraged to exercise regularly as it plays a pivotal role in reducing the incidence or severity of PMS symptoms. With the reduction of the PMS symptoms in the technologists, the provision of services and medical care to patients could also enhance.

Keywords: Premenstrual Syndrome, Operating Room, Occupational Stress

\section{Conflict of Interest: No}

How to Cite: Hannani S, Ghanbary Nekoo N, Nasiri Ziba F, Hosseini AF. The Prevalence of Premenstrual Syndrome and Its Influential Factors in Operating Room Technologists. Iran Journal of Nursing. 2019; 32(118):6777.

Received: 13 March 2019

Accepted: 17 Jun 2019 


\title{
تعيين شيوع سندر ييش از قاعدكى و عوامل مرتبط با آن در تكنولوزيستهاى اتاق عمل
}

\author{
صديقه حنانى'، نسرين قنبرى نكو'، فريبا نصيرى زيبا'، آغا فاطمه حسينىع
}

جكيده

زمينه و هدف: سندرم ييش از قاعدگى يكى از شايعترين اختلالات سنين بارورى است و موجب كاهش سطح كارايى شغلى در كروههاى مختلف حرفه

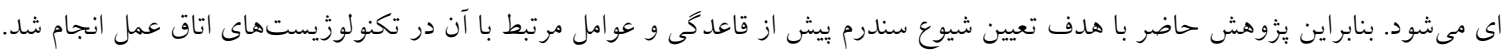

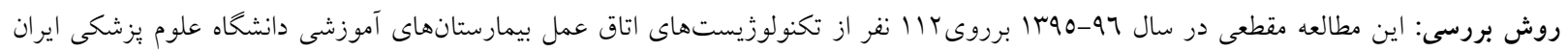

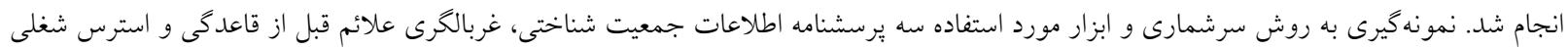

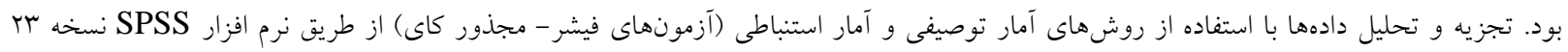

انجام شد.

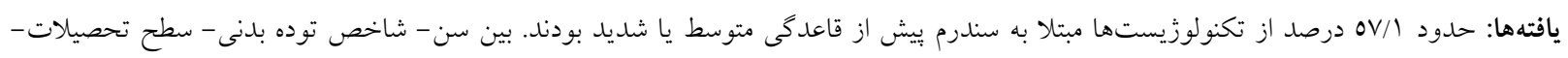

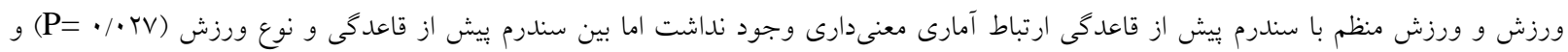

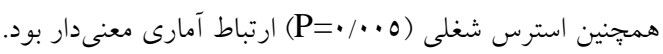

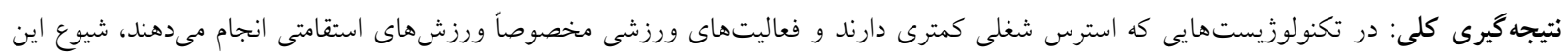

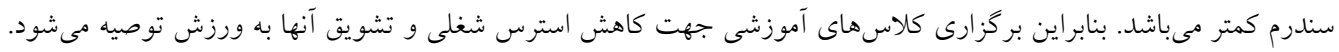

$$
\text { كليد وازهها: سندرم بيش از قاعدگى، اتاق عمل، استرس شغلى }
$$

تاريخ دريافت:

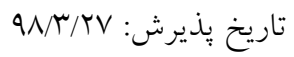

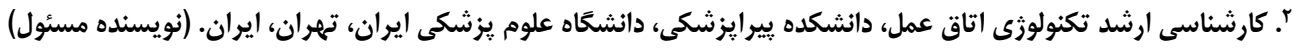


تأثير اين سندرم قرار دارند ولى شايعترين سن ابتلا به اين

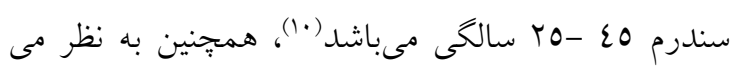
رسد كه افزايش سن نقش مهمى در افزايش علائم اين

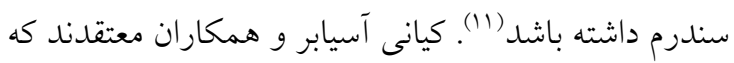
بين PMS و داشتن تمرينات ورزشى منظم يكى رابطه معنى

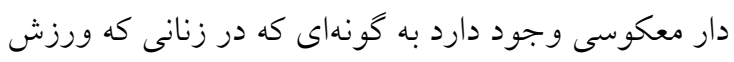

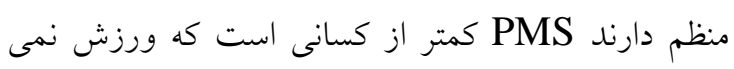

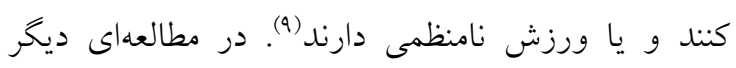

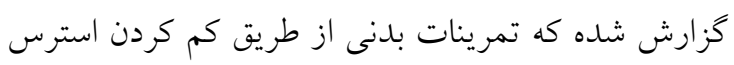

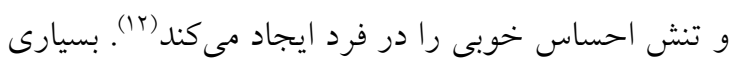

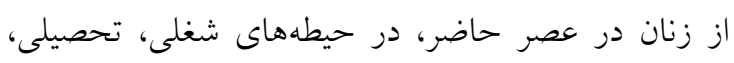

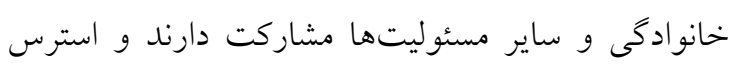

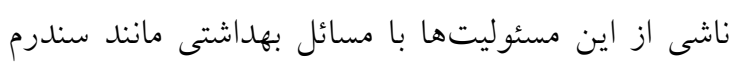

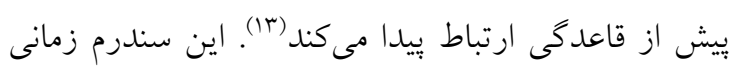

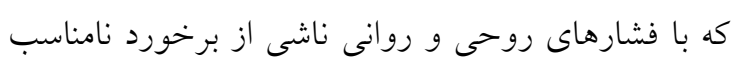

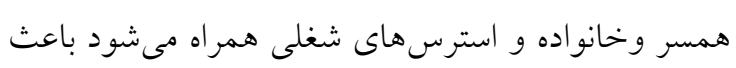

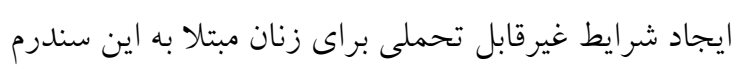

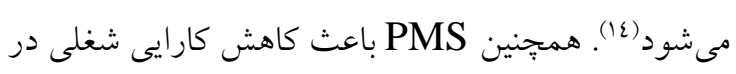

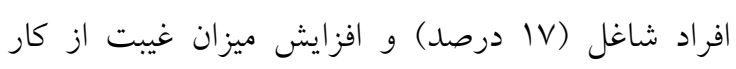
(9/29رصد)(10) و كاهش دقت در انجام كارها و نيز فقدان

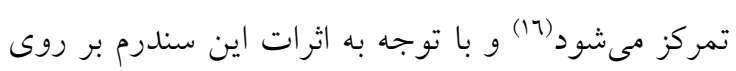

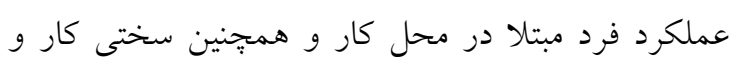

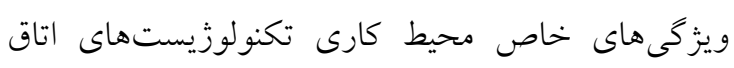

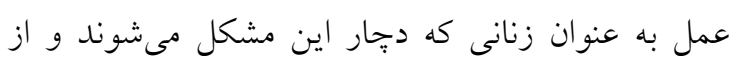

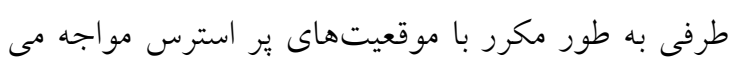

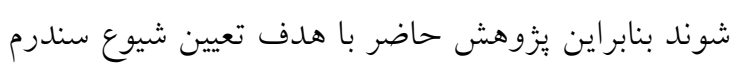

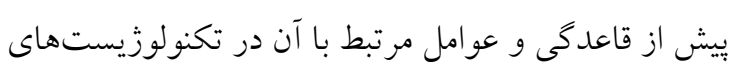

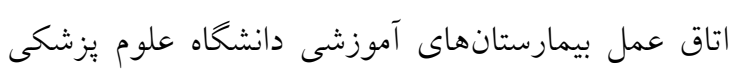

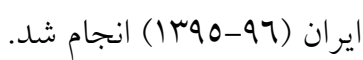

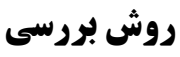

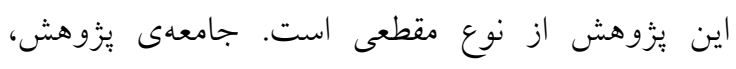

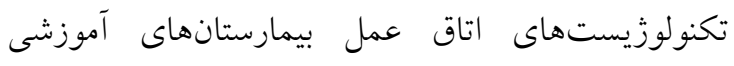

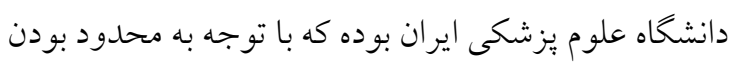

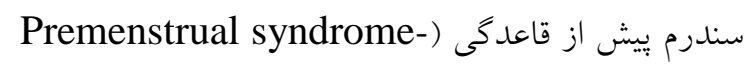

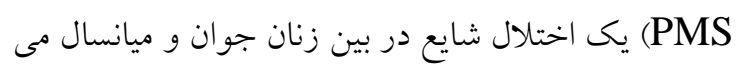

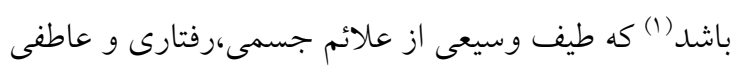

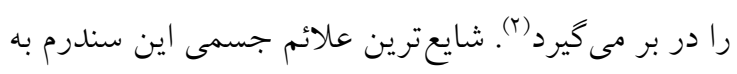

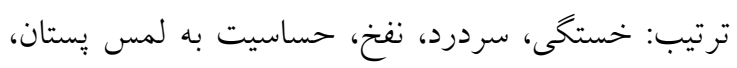

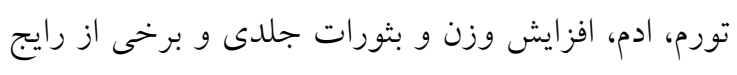

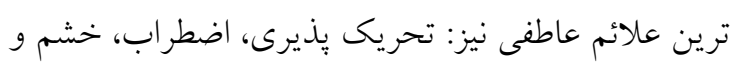

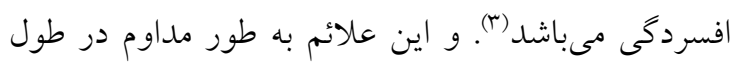

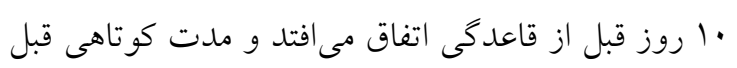

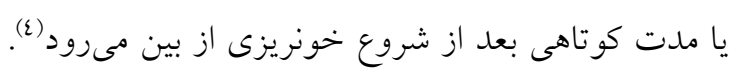

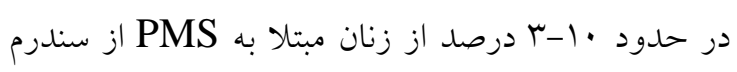
شديد همراه با يريشانى قابل توجه يا اختلال عملكرد رنج

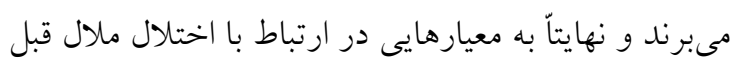
از قاعدىى (Premenstrual Dysphoric -PMDD) مئرسند. disorder

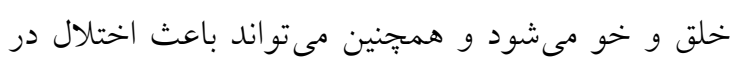

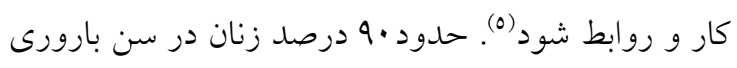

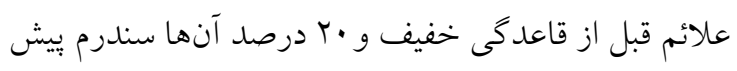

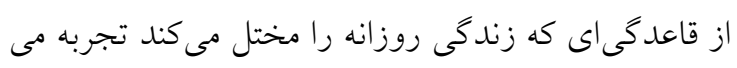

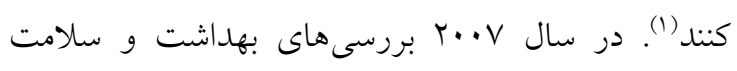

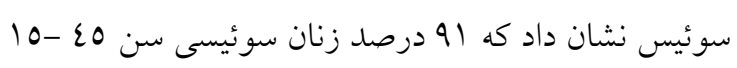

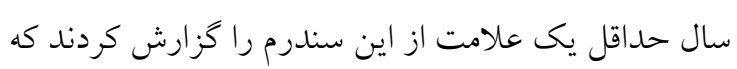

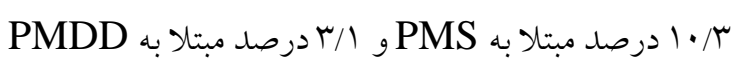

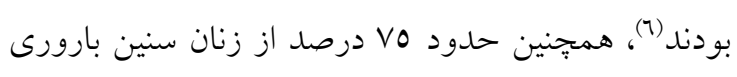

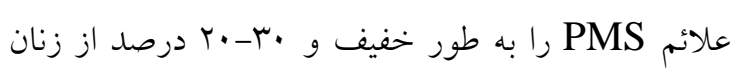

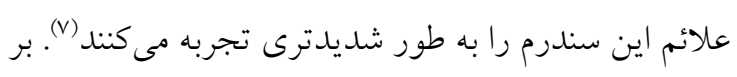

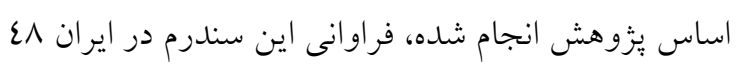

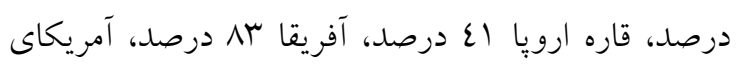

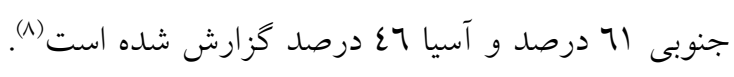

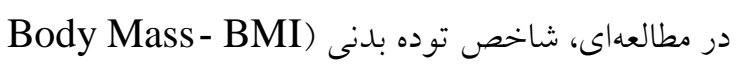

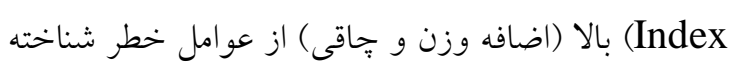

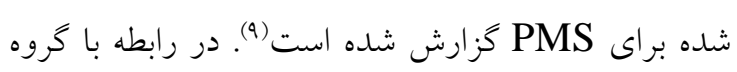

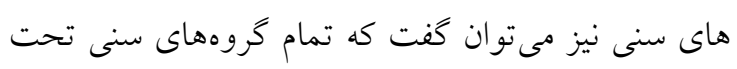


نمرات بالاى 0ح باشد، ميزان استرس شغلى بالا در نظر

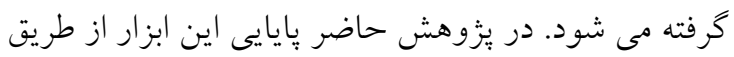

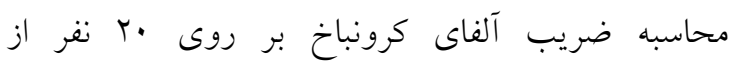

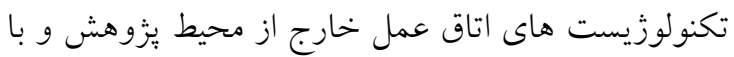

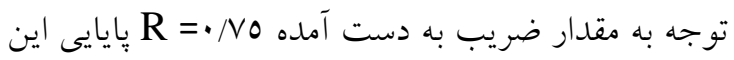

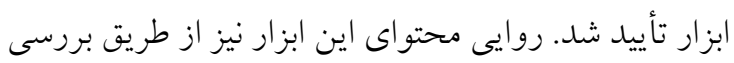

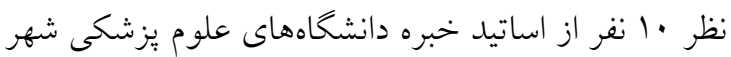

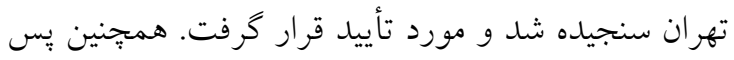

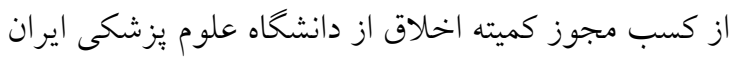
(IR.IUMS.REC 1395.9413101005) معرفى نامه و يس از كسب اجازه از مسئولين واحدهاى يزوهشى و دريافت رضايت آكاهانه از شركت كنندكان،

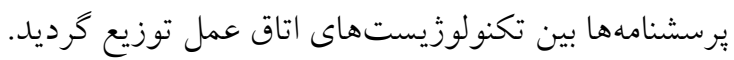
هِ از تكميل و جمع آورى يرسشنامهها به طور كامل، داده

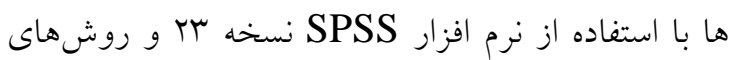

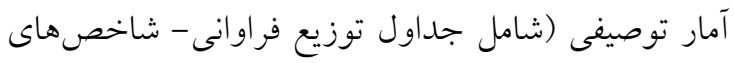

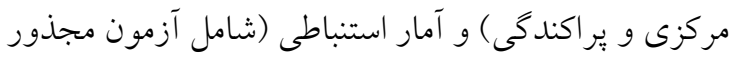

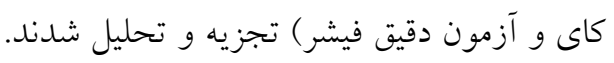

\section{يافتهها}

سن تكنولوزيستهاى اتاق عمل شركت كننده در اين

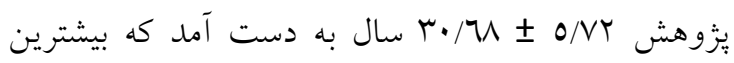
درصد فراوانى مربوط به گروه سنى

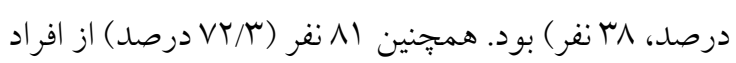

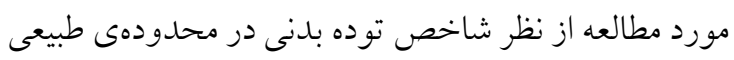

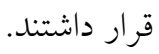

از بين تكنولوزيستهاى مورد مطالعه تنها سب درصد آنها

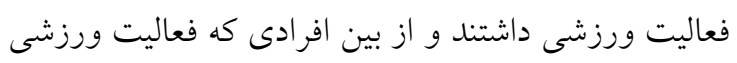

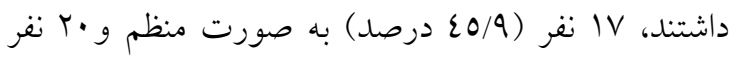
(1/1 ع درصد ) به صورت نامنظم ورزش مى كردند. هم:جنين طبق جدول شماره ا، بيشتر تكنولوزيستها (N// دع درصد) فعاليت ورزشى مقاومتى داشتند. در جامعه مورد يزّوهش •0 درصد افر اد مورد مطالعه داراى استرس شغلى زياد بودند. همجنين ع7 نقر (OV/I درصد)
جامعه، تمامى جامعه يُزوهش به روش سرشمارى وارد

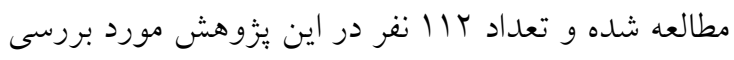

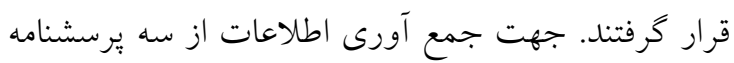

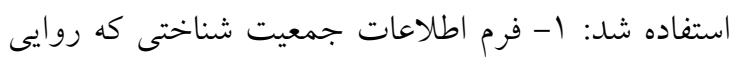

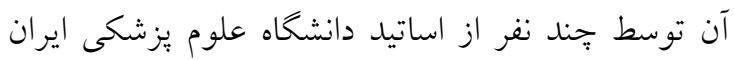

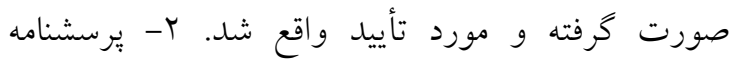

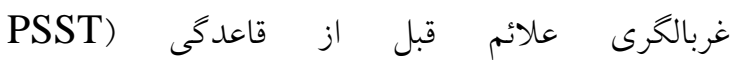
(Premenstrual Symptoms Screening Tool كه سيه بازى و همكاران در سال •وسا در يزّوهش خود

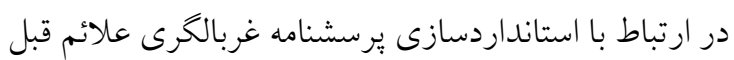

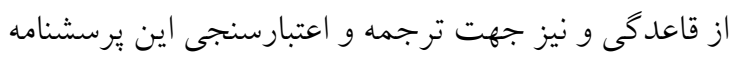

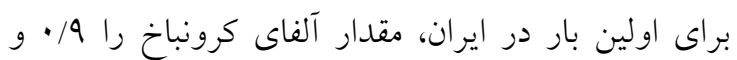

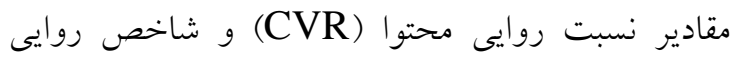

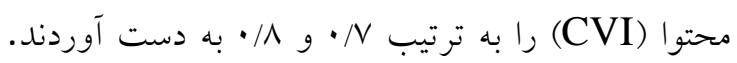

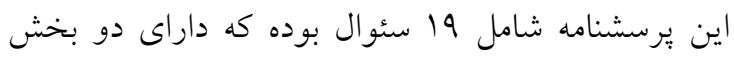

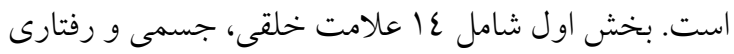
است و بخش دوم نيز تأثير اين علائم را بر زندكى افراد ادئ

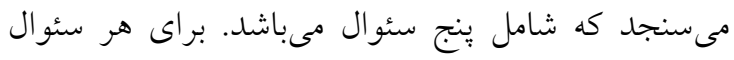

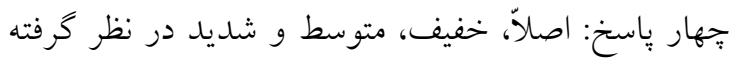

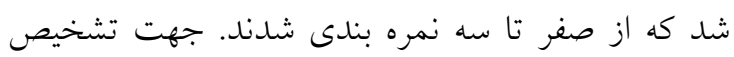

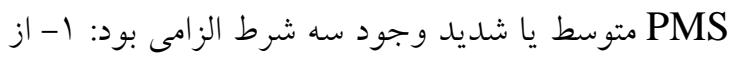

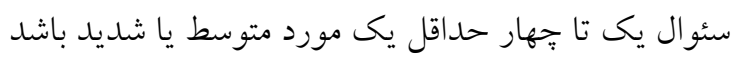

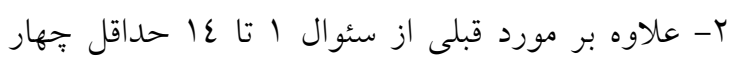

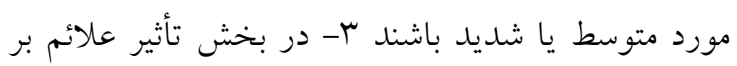

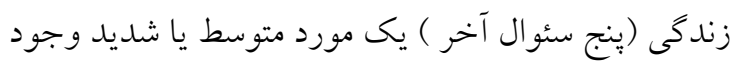

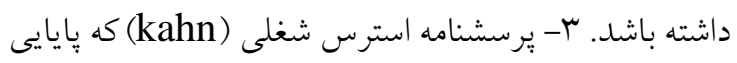

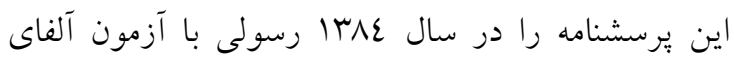

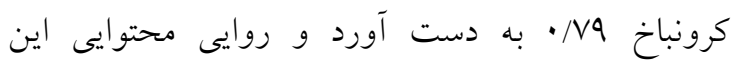
يرسشنامه نيز با استفاده از نظرات اساتيد راهنما و مشاور

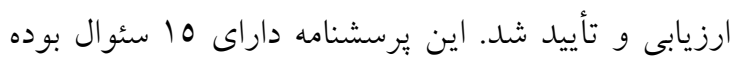

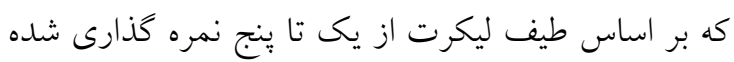

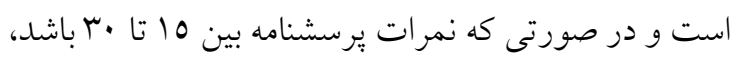

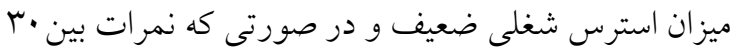
تا مع باشد، ميزان استرش شغلى متوسط و در صورتى كه اله 
از افراد مورد مطالعه داراى PMS متوسط يا شديد بودند

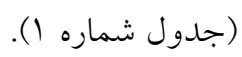

جدول شماره ا: توزيع فراوانى متغيرها در تكنولوزيستهاى اتاق عمل مورد مطالعه سال 97-هوسا

\begin{tabular}{|c|c|c|c|}
\hline درصد & تعداد & سطوح متغير & متغير \\
\hline $1 \pi / \varepsilon$ & 10 & $<r_{0}$ & \\
\hline$r \pi / 9$ & rᄉ & ro-rq & \\
\hline ro & 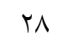 & $r \cdot-r \varepsilon$ & سن \\
\hline $1 / 1 /$ & $r 1$ & ro-ra & \\
\hline $1 / 9$ & 1. & $\geq \varepsilon$. & \\
\hline$\varepsilon / 0$ & 0 & 11//0> كم وزن & \\
\hline$V T / r$ & 11 & 1/1/0-10 طبيعى & $\left(\mathrm{kg} / \mathrm{m}^{2}\right) \mathrm{BMI}$ \\
\hline $19 / 7$ & Tr & •r"ro-r اضافه وزن & \\
\hline$r / 7$ & $\varepsilon$ & P & \\
\hline س & rV & بله & ورزش \\
\hline $7 V$ & vo & خير & \\
\hline$\varepsilon \pi / T$ & 17 & استقامتى & \\
\hline$\varepsilon \Lambda /\rceil$ & 11 & 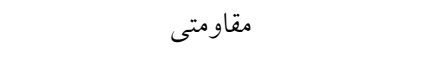 & 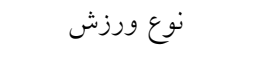 \\
\hline N/1 & $r$ & هر دو & \\
\hline$\varepsilon / 0$ & 0 & كم & \\
\hline$\varepsilon 0 / 0$ & 01 & 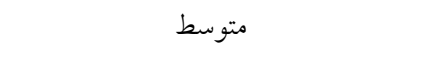 & 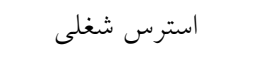 \\
\hline 0. & 07 & زياد & \\
\hline$\sum r / 9$ & $\varepsilon \wedge$ & بدون سندرم ييش از قاعدگى يا خفيف & سندرم ييش از قاعدكى \\
\hline$O V / 1$ & $7 \varepsilon$ & متوسط يا شديد & \\
\hline
\end{tabular}

دادند كمتر از كسانى بود كه ورزش مقاومتى يا هر دو نوع ورزش را با هم انجام مى دهند (PV = • (PV). بر اساس يافتههاى يزوهش شيوع PMS در افرادى كه به

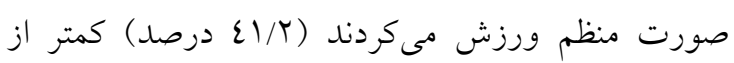
كسانى بود كه به صورت نامنظم فعاليت ورزشى را انجام مى دادند (00 درصد)، هر جند كه از لحاظ آمارى اين ارتباط

$$
\text { معنى دار نبود (P= (P) }
$$

همانطور كه جدول شماره r نشان مىدهد، بين PMS و استرس شغلى ارتباط آمارى معنىدارى وجود داشت به همون طورى كه با افزايش استرس شغلى درصد ابتلا به PMS

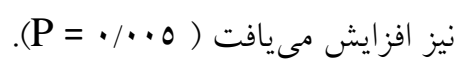

بر اساس نتايج داده ها بيشترين ميزان شيوع PMS در افراد

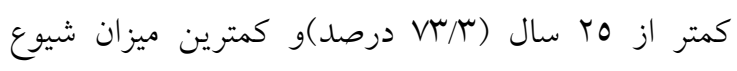
PMS

$$
(\mathrm{P}=\cdot / 10 \varepsilon)
$$

در بررسى ارتباط بين PMS با شاخص توده بلدنى بيشترين ميزان شيوع PMS در افراد داراى اضافه وزن (T/T7 درصد) ديده شد ( (911) • (P=). همجنين شيوع PMS در افرادى كه فعاليت ورزشى نداشتند بيشتر از كسانى بود كه ورزش مى كردند اما از لحاظ آمارى اين اختلاف معنىدار

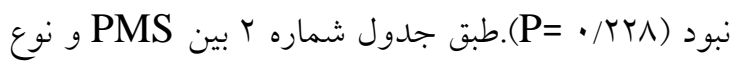
ورزش اختلاف آمارى معنىدارى وجود داشت به طورى كه شيوع PMS در كسانى كه ورزش استقامتى انجام مى 
جدول شماره r: توزيع فراوانى سندرم بيش از قاعدتى بر حسب متغيرهاى مورد بزوهش در تكنولوزيستهاى اتاق عمل مورد مطالعه

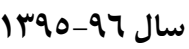

\begin{tabular}{|c|c|c|c|c|c|c|}
\hline \multirow[t]{2}{*}{ سطح معنى دارى } & \multicolumn{2}{|c|}{ ابتلا به سندرم ييش از } & \multicolumn{2}{|c|}{ عدم ابتلا به سندرم بيش از } & \multirow[t]{2}{*}{ سطوح متغير } & \multirow[t]{2}{*}{ متغير } \\
\hline & درصد & فراوانى & درصد & فراوانى & & \\
\hline & $V \pi /$ & 11 & $r T / V$ & $\varepsilon$ & $<r_{0}$ & \\
\hline & $\pi / \pi$ & $r \varepsilon$ & $\mathrm{r} / \mathrm{s}$ & $1 \varepsilon$ & ro-rq & \\
\hline$\# \mathrm{P}=\cdot / 10 \varepsilon$ & $\varepsilon \tau / \varepsilon$ & ir & $0 r / 7$ & 10 & $r \cdot-r_{\varepsilon}$ & سن \\
\hline \multirow[t]{3}{*}{ Value $=\urcorner / \neg \uparrow r$} & $71 / 9$ & ir & $r_{N / 1}$ & $\wedge$ & ro-ra & \\
\hline & $r$. & r & v. & v & $\geq \varepsilon$. & \\
\hline & 7. & r & $\varepsilon$. & r & 1N/0 >كم وزن & \\
\hline \multirow[t]{3}{*}{$* \mathrm{P}=\cdot / 911$} & $00 / 7$ & 2o & $\varepsilon \varepsilon / \varepsilon$ & m & 1N/0-Y طبيعى & $\left(\mathrm{kg} / \mathrm{m}^{2}\right)$ BMI \\
\hline & $7 \pi / 7$ & $1 \varepsilon$ & $r y / \varepsilon$ & $\wedge$ & •ro_r اضافه وزن & \\
\hline & 0. & r & 0. & r & جاق >r. & \\
\hline$\# \mathrm{P}=\cdot / r \Upsilon \wedge$ & $\varepsilon N / \tau$ & 11 & $01 / \varepsilon$ & 19 & بله & ورزش \\
\hline Value $=1 / 7 \uparrow \Lambda$ & $71 / \pi$ & $\varepsilon 7$ & $r / N$ & rq & خير & \\
\hline \multirow[t]{3}{*}{$* \mathrm{P}=\cdot / \cdot T V$} & ro & $\varepsilon$ & vo & rr & استقامتى & \\
\hline & $77 / \mathrm{V}$ & ir & $r \mu / r$ & 7 & مقاومتى & نوع ورزش \\
\hline & $77 / \mathrm{V}$ & r & $r \mu / \mu$ & 1 & هر دو & \\
\hline \multirow[t]{3}{*}{$* \mathrm{P}=\cdot / \cdot \cdot 0$} & ro & 1 & vo & $r$. & كم & \\
\hline & kq & r & $\Delta F$ & $T V$ & متوسط & استرس شغلى \\
\hline & $V T / V$ & r. & $T V / r$ & 10 & زياد & \\
\hline
\end{tabular}

نتايجى متفاوت با يزوهش حاضر دست يافتند به كونهاى بحث و نتيجه كيرى

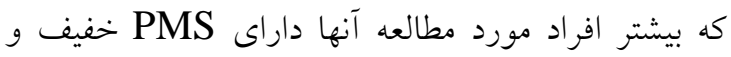

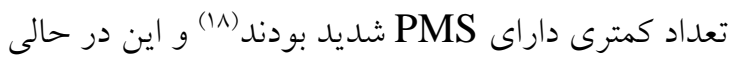
است كه بر اساس بُزوهشهايى كه قبلاً انجام شده، اتاق داق دان عمل يك واحد بيمارستانى است كه با استرس زياد شناخته

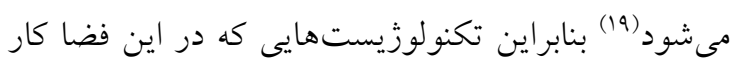
مى كنند داراى استرس شغلى زيادى هستند كه اين امر نيز

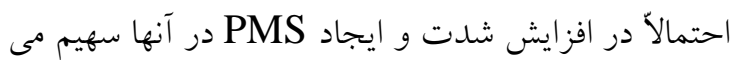

طبق بزوهش حاضر بيشترين ميزان شيوع PMS در افراد

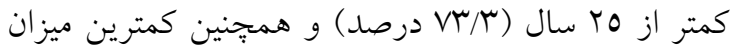

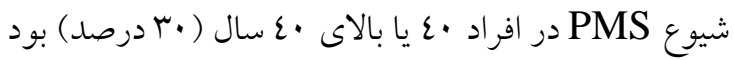

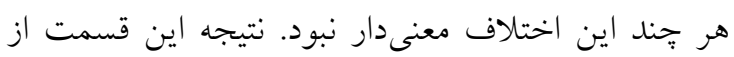
يزوهش با نتايج برخى از مطالعات همخوانى دارد خينانجه

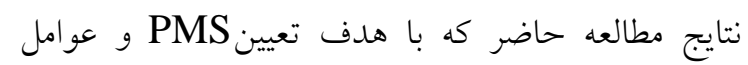
مربوطه در تكنولوزيستهاى اتاق عمل بيمارستانهاى

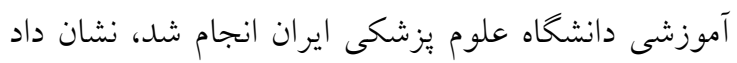
كه بيشتر افراد مورد يُزوهش داراى PMS متوسط تا شديد هستند ( OV/I درصد) در صورتى كه تعداد كمترى از افراد

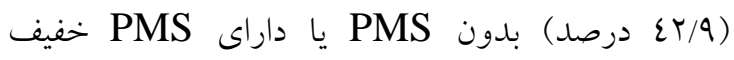

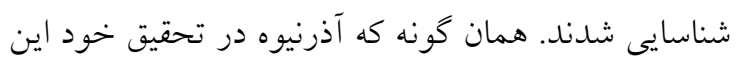

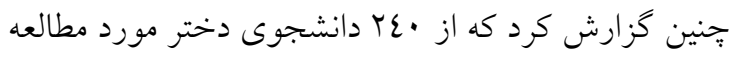

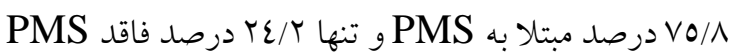
بودند كه اين نيز نشان دهندهى شيوع بالاى PMS در بين بين

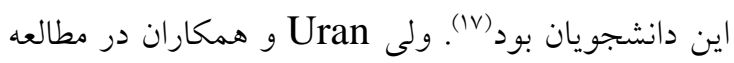
خود برروى 00 نفر از نوجوانان سن سيزده تا هجده سال كه در بخش روانيزشكى دانشخاه آنكارا بسترى بودند، به لهيه 
اضافه وزن بيشتر ديده شده است. بر اساس يزوهش حاضر،

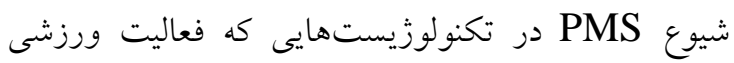

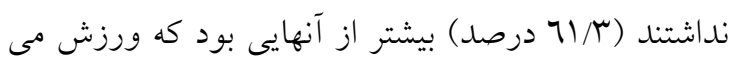

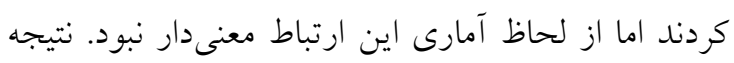
اين بخش از يزوهش مشابه برخى تحقيقات ديخر مى باشد.

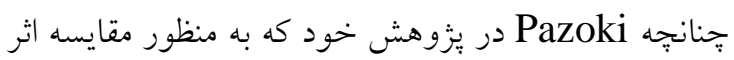

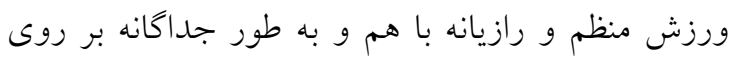

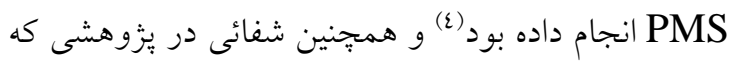
با هدف مقايسه فراوانى اختلالات قاعدكى (آمنوره-

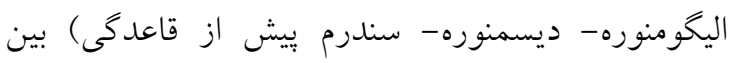
دانشجويان ورزشكار و غير ورزشكار انجام داده بود به اين

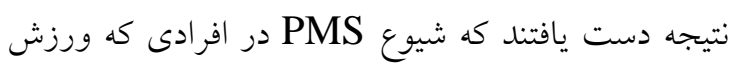

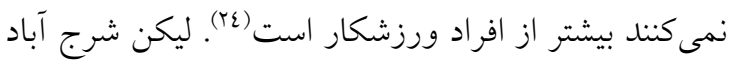
در تحقيقى كه به منظور تعيين فراوانى PMS و ارتباط آن

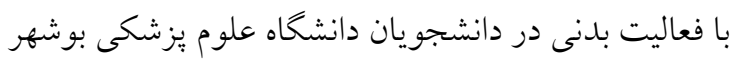

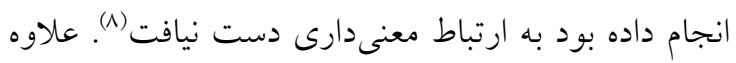

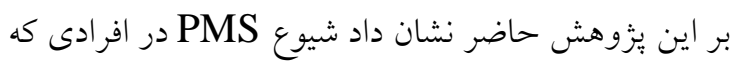

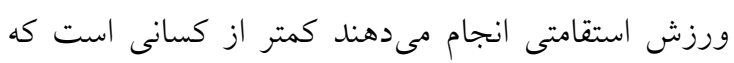
ورزش مقاومتى يا هر دو نوع ورزش را إ انجام مىدهند.

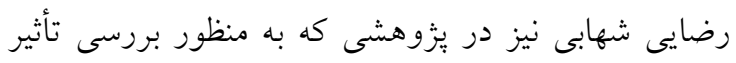

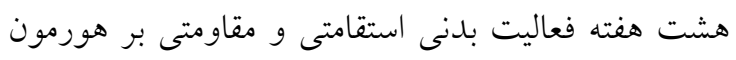
هاى جنسى و علائم PMS در دانشجويان غير ورزشكار

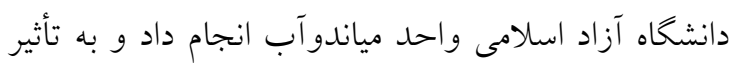
مثبت دو ماه فعاليت ورزشى استقامتى در مقايسه با فعاليت

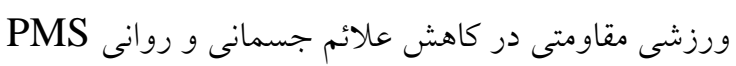

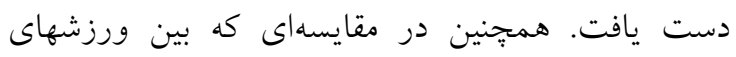

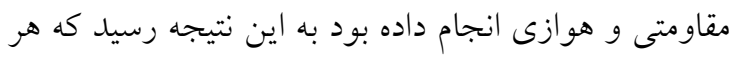
دو فعاليت مقاومتى و هوازى موجب كاهش علائم PMS

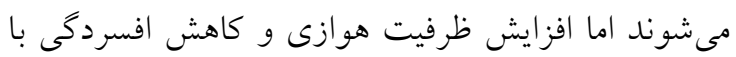
تمرينات هوازى جشمخيرتر از تمرينات مقاومتى مى

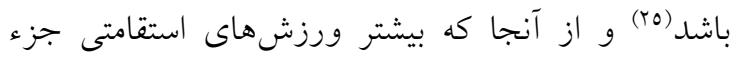
ورزش هاى هوازى نيز محسوب مىشوند بنابر اين اين مورد

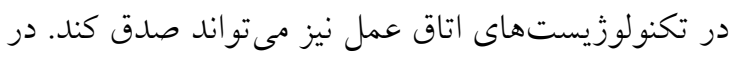

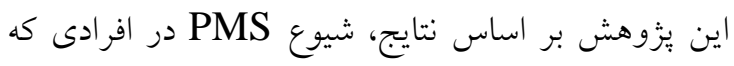

Taneepanichskul و Prungsin دادند كه افراد با سن كمتر بيشتر از PMS رنج مىبرند (•r).

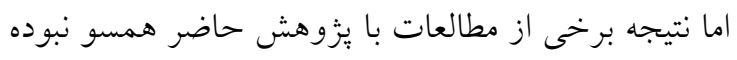

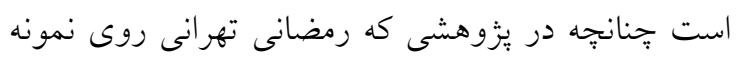

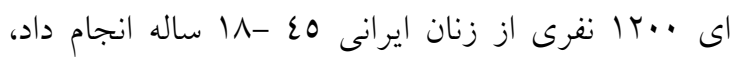

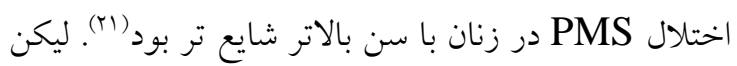

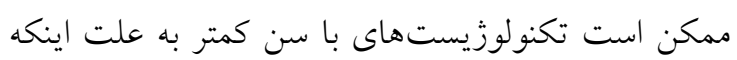

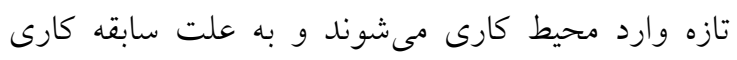

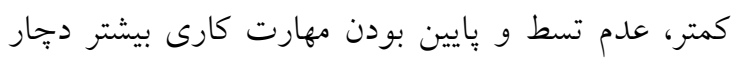

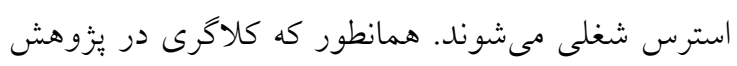
خود به وجود رابطه معنىدار معكوس بين سن و ميانكين

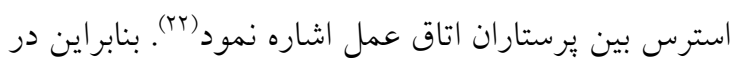
تكنولوزيستهاى با سن كمتر ميزان استرس و در نتيجه

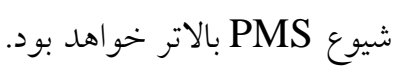

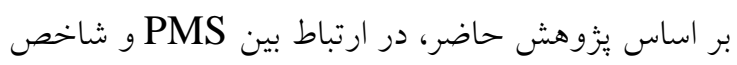

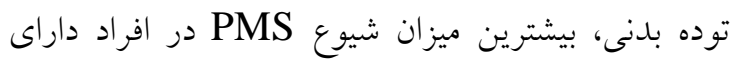

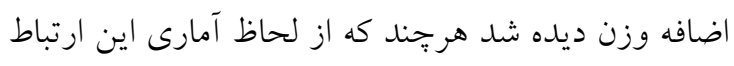

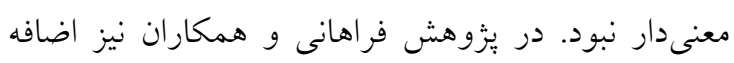

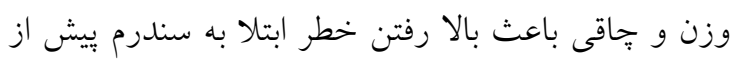

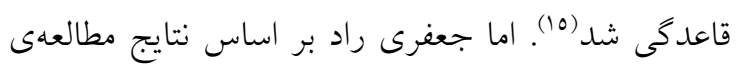

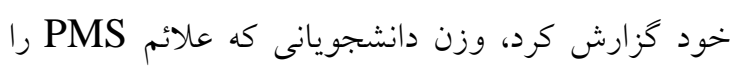

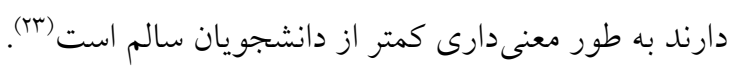

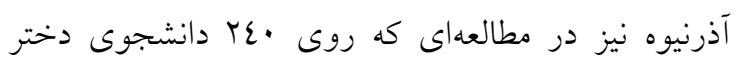

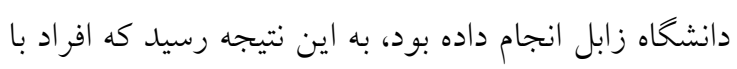

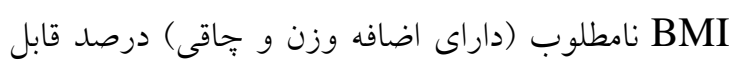

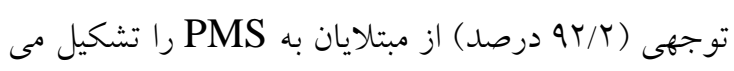
دهند كه اين نيز نشان دهندهى شيوع بالاى PMS در افر افراد داراى اضافه وزن و جّاق نسبت به دانشجويان لاغر بود (IV).

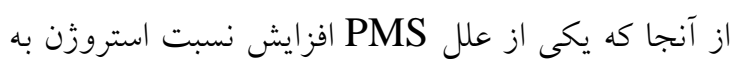
يروزسترون مىباشد و در تكنولوزيستهاى داراى اضافه لئه

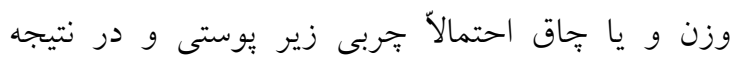
استروزن بيشترى در اين افراد وجود داشته،بنابراين به طور كلى شيوع PMS نيز در اين افراد بيشتر ديده مى إنود

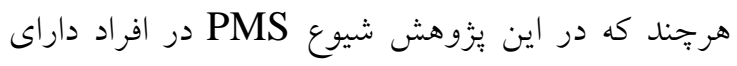


معكوسى داشته (Tr) بنابراين به نظر مىرسد استرس شغلى

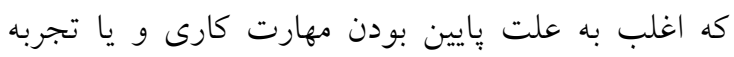

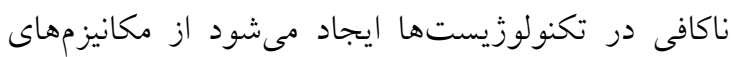
مؤثر بر ايجاد علائم روانى و خلقى باشد. همجنين با توجه به اهميت كيفيت شغلى تكنولوزيستهاى اتاق عمل و نقش مهم آنان در تيم جراحى، اجر اي برنامههاى بيشكير انه

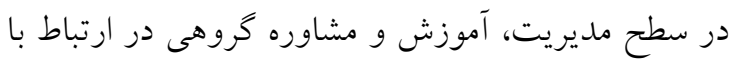

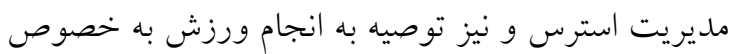
ورزشهاى استقامتى به صورت سازمانى مىتواند سهم

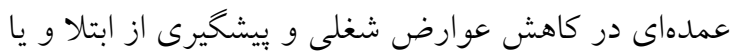
كاهش علائم PMS داشته باشد و به دنبال كاهش علائم PMS و نيز كاهش ميزان استرس شغلى تكنولوزيستها،

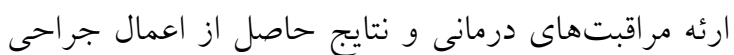
نيز مطلوبتر خواهد بود مرابت دماني

تعارض منافع: هيج گونه تعارض منافع از سوى نويسندگان بيان نشده است.

\section{تقدير و تشكر}

اين ثيزوهش برگرفته از بِايان نامه كارشناسى ارشد اتاق

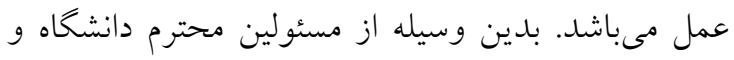

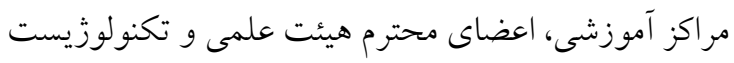
هاى خدوم اتاق عمل بيمارستانهاى آموزشى دانشخاه علوم

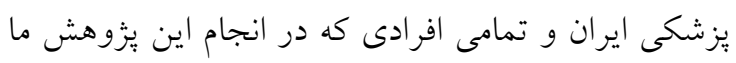
را يارى نمودند، تشكر و قدردانى مى گرددا.
ورزش منظم انجام مىدهند كمتر از كسانى است كه ورزش

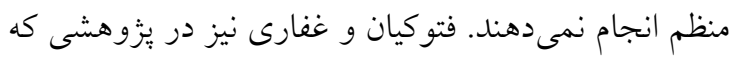
به منظور تعيين تأثير يك برنامه ورزشى منظم هوازى كروهى بر شدت سندرم قبل از قاعدگى انجام داده بودند

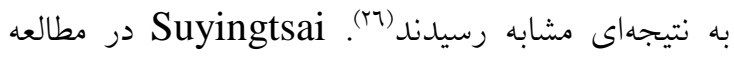
خود نشان داد كه ورزش منظم باعث كاهش درد قاعدى مئى

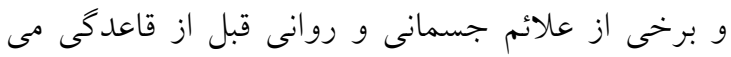

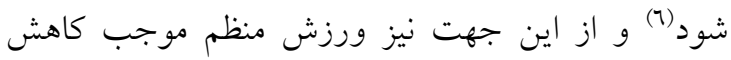

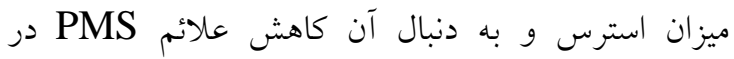
تكنولوزيستهاى اتاق عمل مى شود. در ارتباط با استرس شغلى و PMS نتايج يُزوهش حاضر نشان داد كه ارتباط

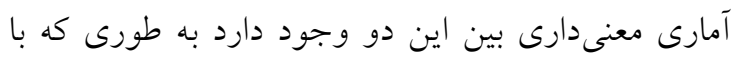

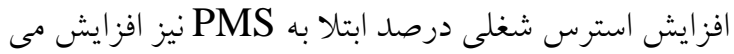

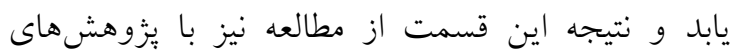

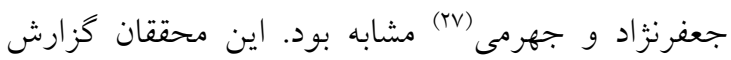
كردند افرادى كه استرس كمترى دارند، شدت PMS

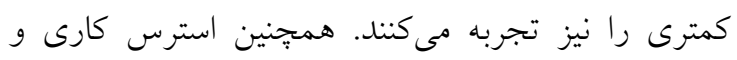

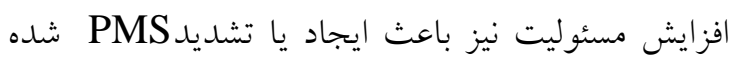
بود2 (rN) از محدوديتهاى اين مطالعه مىتوان به عدم بررسى تعداد

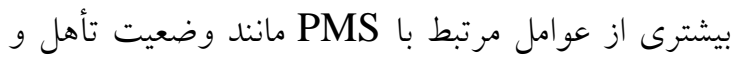
سطح تحصيلات و ديخرى محدود بودن جامعه اشاره نمود كه ييشنهاد مىشود مطالعات بيشترى در ارتباط با عوامل

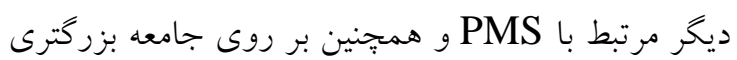
از تكنولوزيستها، صورت كيرد.

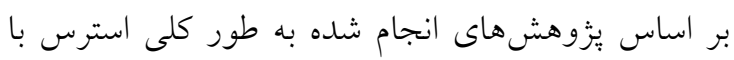
سن و سابقه كارى يرسنل اتاق عمل رابطه معنىدار

\section{References}

1- Ducasse D, Jaussent I, Olié E, Guillaume S, Lopez-Castroman J, Courtet P. Personality traits of suicidality are associated with premenstrual syndrome and premenstrual dysphoric disorder in a suicidal women sample. PloS one. 2016;11(2):e0148653.

2- Kumari S, Sachdeva A. Patterns and Predictors of Premenstrual Symptoms among Females Working in a Psychiatry Hospital. Scientifica. 2016;2016.

3- Beiranvand SP, Beiranvand NS, Moghadam ZB, Birjandi M, Azhari S, Rezaei E, Salehnia AN, Beiranvand $\mathrm{S}$. The effect of Crocus sativus (saffron) on the severity of premenstrual syndrome. Eur J Integr Med. 2016;8(1):55-61. 
4- Pazoki H, Bolouri G, Farokhi F, Azerbayjani MA. Comparing the effects of aerobic exercise and Foeniculum vulgare on pre-menstrual syndrome. Middle East Fertil Soc J. 2016;21(1):61-4.

5- Brahmbhatt S, Sattigeri BM, Shah H, Kumar A, Parikh D. A prospective survey study on premenstrual syndrome in young and middle aged women with an emphasis on its management. Int J Res Med Sci. 2013;1(2):69-72.

6- Tsai SY. Effect of yoga exercise on premenstrual symptoms among female employees in Taiwan. Int J Environ Res Public Health. 2016;13(7):721.

7- Abd Nejad R, Simbar M, Sheikhan Z,Mogab F,Nasiri M. The effect of salivary extract on the psychological symptoms of premenstrual syndrome . IJOGI .2017;20(10):84-94. [Persian]

8- Najafi-Sharjabad F, Borazjani F, Avazzadeh Z. Investigation of prevalence and severity of premenstrual syndrome and its relationship with exercise among female students in Bushehr University of Medical Sciences. Pajouhan Scientific Journal. 2017;15(3):43-50. [Persian]

9- Sahin S, Ozdemir K, Unsal A. Evaluation of premenstrual syndrome and quality of life in university students. J Pak Med Assoc. 2014;64(8):915-22.

10- 10-Jamali S, Karimi F. Comparison of Premenstrual Syndrome in Unmarried and Married Women in West Ivan City of the Year (2013). J Kermanshah Univ Med Sci . 2012;18( 10):593-98. [Persian]

11- Armand A, Talaei A. The effectiveness of cognitive behavioral stress management training in reducing psychological problems and symptoms of premenstrual syndrome. Iran J Obstet Gynecol Infertil. 2012;15:21-61.; 15(21): 24-31. [Persian]

12- Moghadasi A, Abbasi M, Yousefi M, Kargarfard M. A comparison of prevalence of premenstrual syndrome symptoms between athlete and non-athlete female students. J Sports Physiol Act. 2009;3:199-208. [Persian]

13- Zarei Z, Bazzazian S. The relationship between premenstrual syndrome disorder, stress and quality of life in female students. Iranian Journal of Psychiatric Nursing. 2015;2(4):49-58. [Persian]

14- Akbari MA, Mohammadrezayi J, Khodadadi E, Naderi J. Nurses'experiences Of Family Problems Caused By Premenstrual Syndrome: A Phenomenological Study. The J Urmia Nurs Midwifery Fac. 2016;14(5):474-84. [Persian]

15- Farahani LA, Heidari T, Narenji F, Jafarabadi MA, Shirazi V. Relationship between pre menstrual syndrome with body mass index among university students. Hayat. 2011;17(4):85-95. [Persian]

16- Shobeiri F, Ezzati Arasteh F, Ebrahimi R, Nazari M. Effect of calcium on physical symptoms of premenstrual syndrome. The Iranian Journal of Obstetrics, Gynecology and Infertility. 2016;19(1.2):1-8. [Persian]

17- Azarnive MS, Tavakoli KS. Level of Physical Activity and Pre Menstrual Syndrome amongst Female University Students. Avicenna Journal of Nursing And Midwifery Care (Scientific Journal of Hamadan Nursing \& Midwifery Faculty). 2016;24(2): 68-75. [Persian]

18- Uran P, Yürümez E, Aysev A, Kılıç BG. Premenstrual syndrome health-related quality of life and psychiatric comorbidity in a clinical adolescent sample: a cross-sectional study. Int J Psychiatry Clin Pract. 2017;21(1):36-40.

19- Chen CK, Lin C, Wang SH, Hou TH. A study of job stress, stress coping strategies, and job satisfaction for nurses working in middle-level hospital operating rooms. Journal of Nursing Research. 2009;17(3):199-211.

20- Prungsin T, Taneepanichskul S. Prevalence and Quality of Life (QOL) with Premenstrual Syndrome (PMS) among the Working Women in Reproductive Age Group in Bangkok, Thailand. Journal of Health Research. 2016;30(Suppl. 2):S139-45.

21- Ramezani Tehra F, Robab Allameh M. Prevalence of premenstrual syndrome and some of its relative factors in reproductive age. The Horizon of Medical Sciences. 2012;18(3):121-7.

22- Kolakari SH, Sanakoo A, Mirkarimei F, Behnampour N. The level of stress among Gorgan University of Medical Sciences hospital operation room's personals and its relation to some related factors. Journal of Gorgan University of Medical Sciences. 2002;4(10):54-59. [Persian]

23- 23 Jafarirad S, Rasaie N, Darabi F. Comparison of Anthropometric Indices and Lifestyle Factors between Healthy University Students and Those Affected by Premenstrual Syndrome. Jundishapur Scientific Medical Journal. 2016; 15(2): 217-27. [Persian]

24- Sehati Shafaie F, Matin Homaei H, Zoodfekr L. Comparison the frequency of menstrual disorders (amenorrhea, oligomenorrhea, dysmenorrhea and premenstrual syndrome) between athletes and 
non-athletes female students of Tabriz universities, Tabriz, Iran. The Iranian Journal of Obstetrics, Gynecology and Infertility. 2013;16(51):14-21. [Persian]

25- Rezaei Shahabi Z, Ebrahim KH,Gholami M. The Effect of 8-Week Endurance and Resistance Physical Activity on Sex Hormones and Signs of Premenstrual Syndrome in Non-Athlete Students .Q J Sport Life Sci. 2011;2(5):65-73. [Persian]

26- Fotokian Z, Ghaffari F. Aerobic exercise program on the intensity of premenstrual syndrome. Iran J Dermatol. 2006;8(4):76-80. [Persian]

27- Jafarnejad F, Shakeri Z, Najaf Najafi M, Salehi Fadardi J. Evaluation the relationship between stress and the risk of premenstrual syndrome. The Iranian Journal of Obstetrics, Gynecology and Infertility. 2013;16(76):11-8. [Persian]

28- Jahromi BN, Pakmehr S, Hagh-Shenas H. Work stress, premenstrual syndrome and dysphoric disorder: are there any associations?. Iranian red crescent medical journal. 2011;13(3):199. [Persian] 